\section{FURTHER LIGHT UPON THE NATURE OF} THE BENZENE NUCLEUS.

$A \mathrm{~N}$ important memoir, containing an admirable compendium of the data now accumulated bearing upon the much-discussed question of the nature of the fundamental hydrocarbon of the aromatic compounds, together with the results of new spectrometric observations of great value, is contributed to the current issue of the Fournal fiur praktische Chemie, by Prof. J. W. Bribl, of Heidelberg. The main question now at issue is whether benzene is best represented by the well-known structural formula of Keliulé,<smiles>c1ccccc1</smiles>

in which the carbon atoms are linked together by alternate double and single linkages, or by a formula in which there are no double linkages, and each carbon atom is attached to three others, the three extra linkages being diagonal,

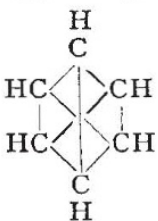

a view which has latterly received some support at the hands of Prof. von Baeyer. A full discussion of the valuable experimental work of the latter chemist is given, together with the thermochemical work of Thomsen, Dieffenbach, Horstmann and Stohmann.

Prof. Briihl has recently determined the specific gravities and optical constants of the three compounds benzene dihydride $\mathrm{C}_{6} \mathrm{H}_{8}$, benzene tetrabydride $\mathrm{C}_{6} \mathrm{H}_{10}$, and benzene hexahydride $\mathrm{C}_{6} \mathrm{H}_{10}$, the two former of which were prepared some time ago by Prof. von Baeyer, together with those of hexylene. 'There havenow been fully investigated as regards the spectrometric constants eight compounds which are so closely related as to enable most important deductions to be derived from their comparison. These compounds are : benzene itself $\mathrm{C}_{6} \mathrm{H}_{6}$, benzene dihydride $\mathrm{C}_{6} \mathrm{H}_{8}$, benzene tetrahydride $\mathrm{C}_{6} \mathrm{H}_{30}$, benzene hexahydride $\mathrm{C}_{6} \mathrm{H}_{12}$, hexane $\mathrm{C}_{6} \mathrm{H}_{11}$, hexylene $\mathrm{C}_{6} \mathrm{H}_{12}$, diallyl $\mathrm{C}_{6} \mathrm{H}_{10}$, and dipropargyl $\mathrm{C}_{6} \mathrm{H}_{6}$. The second, third, and fourth are the graduated products of the addition of hydrogen to benzene, two atoms at a time; the fifth, hexane, is the open chain six carbon paraffin, hexylene the six carbon olefine with one double linkage, dially the six carbon fatty compound containing two double linkages, and dipropargyl the six carbon compound containing two acetylene triple linkages.

Upon comparing the specific gravities at $20^{\circ}$ of these eight compounds it is observed that the density steadily decreases from benzene to benzene hexahydride; there is then a sudden large fall upon the disruption of the ring and formation of the open chain compound hexane. The density then slightly increases through hexylene and diallyl, and again a sudden break of continuity, a large rise, occurs upon the passage to the acetylene derivative dipropargyl. Still more striking are the changes exhibited by the molecular volumes. There is an exceptionally large rise of twenty-three units between benzene hexahydride and hexane, and a similar large decrease between diallyl and dipropargyl. Passing to the molecular refraction, it is observed that upon the graduated addition of hydrogen to benzene, this constant becomes gradually larger as far as the hexahydride, then there is a great leap upon the breaking of the ring and production of hexane. Similarly as the hydrogen is again removed step by step a continuous decrease occurs unti diallyl is reached, when upon removal of $2 \mathrm{H}_{3}$ and formation of dipropargyl, the two ethylene groups being changed into acetylene radicles, the molecular refraction falls precipitately.

The whole of these physical properties thus exhibit break of continuity on passing from benzene hexahydride to hexane, that is upon the opening of the ring into a straight chain, and also when the ethylene derivatives are converted into derivatives of acetylene. Moreover, if the isomers among these eight compounds are compared, benzene with dipropargyl, benzene tetrahydride with diallyl, and benzene hexahydride with hexylene, it is found that the physical constants differ very materially. From these considerations Prof. Bribl concludes that the Kekulé structural formula is most in accordance with the facts.

In addition to the above new observations, the spectrometric constants of the ethyl ester of phthalic acid have been determined. Prof. von Baeyer considered that he had proved that phthalic acid cannot be constituted according to Kekule's conception of the aromatic nucleus, but that a nucleus with three diagonal single linkages must be present. Upon comparing, however, the observed molecular refraction and dispersion of the ethyl ester with the values for these constants calculated upon the assumptions of the two hypotheses, they are found to correspond closely with those demanded by the Kekulé structural formula, and are very far removed from those calculated upon the basis of three diagonal linkages.

Prof. Briihl considers it to be well founded that in benzene tetrahydride and in hexylene there is one ethylenic double linkage, and that in benzene dihydride and in diallyl there are two such linkages. Now the continuity in the entire physical properties as hydrogen is removed step by step from hexane to diallyl on the one hand, and from benzene hexahydride to benzene itself upon the other, points conclusively to a continuity in the nature of the alteration of the constitution in both series. Benzene must therefore, according to Prof. Brühl, likewise contain ethylenic double linkages, three in number, if benzene dihydride contains two such linkages and the tetrahydride one. The view that three effective diagonal linkages, or, as has recently been surmised by certain chemists, three central potential linkages, can bring about in benzene the same physical action as three ethylenic bonds, appears to Prof. Briibl to be out of the question. He shows, moreover, that the values for the whole of the eight substances agree most remarkably with the numbers calculated upon the basis of the Kekulé formula, and further, that the thermodynamical data all point to the same conclusion.

The relation of the atoms to one another in the benzene nucleus is not, however, ideally expressed by Kekulé's structural formula. This can only be achieved by a spacial representation. The happiest conception of the spacial configuration of benzene, according to Prof. Brubl, is that of Sachse. This model is constructed by taking a cardboard octahedron, removing two parallel sides, and upon each of the six remaining ones placing a regular tetrahedron. The six tetrahedra represent the six carbon atoms, and the hydrogen atoms are supposed to be attached at the six apices. The six carbon atoms then lie in two parallel planes, as do likewise the six hydrogen atoms. The properties of such an arrangement would be such as accord with the observed facts. The gradual addition of hydrogen would cause a regular and continuous movement of the tetrahedra, corresponding with the observed continuity in physical properties. The best representation of this model in one plane is the structural formula of Kekulé.

\section{A. E. Tutton.}

\section{THE FACE OF THE EARTH. ${ }^{1}$}

$\mathrm{A}^{\mathrm{T}}$ the present time we all acknowledge the value of the accepted classification of the relief-forms of the earth's surface in continents and islands, mountain chains, plateaus, plains, \&c. ; into ocean-basins, seas, lakes, and the like. But few of us ask ourselves the very natural questions, "What is the fundamental unit among all these morphological individuals, great and small ? Is there any surface unit existent among them which, like the species of the biologist, once identified, will not only be found to group its individuals rank over rank into the genera, the families, the orders, and the kingdoms of the surface world; but the study of whose life-history and necessary interactions with its fellow-species will eventually afford us some clue to the relationships and the natural classification of the whole?"

This aspect of the subject perhaps excepted, there is probably no theory possible upon the matter of the grouping of the forms of the earth's surface which has not, either as a whole or in part,

' A aper read by Prof. Chas. Lapworth, F.R.S., at the Royal Geographical Society, on April 23.

NO. 1278 , VOL. 49] 
been already suggested by one investigator or another. In the speculative parts of the subject, the names of Werner, Hutton, De Beaumont, Humboldt, Guyot, Lyell, and Peschel stand conspicuous amongst past investigators; and, among those of the present day, Le Conte, Dana, Crosby, Dutton, and Gilbert in America; Heim, Suess, Penck, and Reyer in Germany ; Réclus, De Lapparent, and Bertrand in France ; and the Geikies, Wallace, Murray, Fisher, Reade, and Mill in Britain.

Turning first to the general disposition of the recognisable parts of the terraqueous surface of the globe, the author passed in review a few of the fundamental facts and conclusions worked out by students of the subject, and showed that it had long since been acknowledged that between all the grander forms of the earth's surface there existed a curious correspondence of shape and of size, combined with a mysterious contrast of geographical arrangements or disposition. Next it was discovered that among the minor elements of surface form, the study of geographical homologies showed us that all the recognisable forms of a higher order discernible upon the earth's surface are made up of kind of rhythmic repetition of forms of a lower order possessing in miniature the characteristics of the major forms. These conclusion; had been practically arrived at by the students of the relief of the globe previous to the recent discoveries of the Challenger and other exploring expeditions, but the result of these deep-sea researches were so strange and so unexpected as almost to dwarf, for the time, these earlier ideas into insignificance.

Deep-sea researches showed that former ideas of the similarity of size and form between the surfaces of the land and water areas as such must be relinquished. For the mean height of the land was found to be only one-sixth of the mean depth of the ocean; and the entire volume of the solid lands above the level of the sea was discovered to be only one-fourteenth of the volume of the ocean waters below that level. Further, what was far more startling and far more important, it was found that the shore-lines of the visible continents by no means mark the true edges of the great ocean-basins; the dry lands were ascertained to be merely the undrowned portions of one universal continental plateau, the surface of which sinks at first very gently from the shore-line through a shallow water area many miles across, and then plunges rapidly downwards in a sudden slope to the true or abyssal floor of the ocean. This spreads out as a broad undulating plain some twelve thousand feet and more below the sea-level, and descends even still deeper locally in magnificent lake-like hollows to depths of from twenty thousand to thirty thousand feet. These results gave us for the first time a map of the forms of a region of the earth's surface at least twice as large as that of the whole of the dry lands united together. Nothing so important, from a geographical point of view, has been accomplished since the days of Columbus. It is the discovery of a new world. But in this new world it is plain that if the deductions of the earlier students of the earth's surface are of any value whatever, they must prove to be equally natural and inevitable.

Leaving the discussion of these deep-sea discoveries for a while, the author next summarised the fundamental conclusions arrived at by the geologist. The geologist has discovered that in all dry lands of the earth which he has hitherto investigated in detail, the local surface of the country is composed of the outcropping edges of solid rock-sheets known as the " geological formations." These formations show distinctly, by their composition and by the relics of marine life which they contain, that they were originally laid down as layers of gravel, sand, and mud upon the floor of the sea. In other words, the surface of every geological formation must have constituted, at the date when it was deposited, an integral portion of the submarine relief of the earth's surface of its time. But while it is clear that these formations were laid down bel sw the sea-level and in an approximately horizontal position, they are now found, wherever we can examine them upon the dry lands, usually far above that sea-level; and, instead of being horizontal, the surface of each formation is now found to be typically warped into great undulations like the surface of a folded cloth, or that of a rolling sea.

The undulations or wave-like forms of the surfaces of the geological formations are of all degrees of importance, the smaller waves riding on the backs of the larger ones, like ripples on the backs of the sea-waves. But in spite of the extreme complexity of this arrangement, it is comparatively easy of study, for we find the whole to be made up of endless repetitions of one and the same fundamental unit-namely, an undulation or wave-like form; and the study of the characteristics and life-history of one of these typical undulations gives us, within certain limits, the key to those of all the rest.

Each simple geological undulation consists of two parts, an arch-like rise and a trough-like fall, and these two reciprocal elements are most naturally and conveniently united by the geologist under the single title of the "crust-wave" or "geological fold."

This curious wave-like disposition of the surface of any geological formation is apparent, whether we follow that surface, say, from east to west, or whether we follow it from north to south. So that the present surface of a formation is most simply pictured if we regard it as having been bent up into two sets of undulations, the one set crossing the other at right angles.

With this geological result as a guide, the author returned to the investigation of the main features of the earth's surface. It was pointed out that any straight line drawn completely round the globe from west to east over the earth's surface, either along the equator, the tropici, or along any of the neighbouring parallels, shows a more or less regularly alternating elevation and depression of that surface, of the same general type as the undulations of a geological section. Along these parallels we have three successive elevations, the Americas, Eurafrica, and Asia-Australia; and three intermediate depressions, the ocean-basins of the Atlantic, the Indian Ocean, and the Pacific. That is to say, the broad forms of these surface undulations naturally suggest the theory that the ex. terior parts of the earth-crust are bent into three primary meridional waves ranging practically from pole to pole, each wave consisting of a single rise and a single fall. Again, if the crest of any one of the three meridional continental ridges is followed in a transverse direction, i.e. from north to south, it is found that the surface of the crest itself rises and falls in its turn in three successive undulations. For example, in the case of the ridge of the Americas, it is crossed by the three transverse ridges of North America, South America, an 1 the Antarctic continent; and the three transverse depressions of the Arctic Ocean, the Carribbean, and the depression south of Cape IIorn.

Thus (precisely as in the case of the surface of a geological formation) the surface of the earth-crust at the present day is most simply regarded as the surface of a continuous sheet which has been warped up by two sets of undulations crossing each other at right angles. But in the case of the earth-surface, the one set ranges parallel with the equator, and the other ranges from pole to pole.

By means of a figure giving the natural disposition of the resultant forms and nodal lines characteristic of the surface of an elastic film warped by two orthogonal and simultaneous sets of undulations, the author showed how the phenomena apparent upon this cross undulated film suggested at a glance, (I) the forms and disposition of the terrestrial continents; (2) the triangular shapes of their extremities; (3) the diagonal treads of their shores ; and (4) the courses of the archipelagic lines.

Carrying the method a stage farther, and breaking up each of the major waves symmetrically in a corresponding manner, the author showed how the subordinate forms so obtained now suggested the typical vertical contour of continents, namely a plain bounded by two marginal ridges and that of an ocean-floor, a submerged plain warped up centrally by a submarine elevation. The same correspondence was shown to hold good even in the broadest grouping of the forms, the collective land, and $w$ ter areas.

After indicating that we have here the hint that the fundamental unit of form of the earth's surface is the wave or fold, and that the surface-contours of the globe are primarily the resultants of the two sets of undulations into which the outer parts of the earth-crust are warped up, the author pointed out that these results up to this point were reliable only as generalities, but appear at first sight valueless when we descend to particulars, and he next endeavoured to explain how the known minor variations and anomalies might be perhaps accounted for.

Returning to the subject of the geological fold, he showed how the various forms of the geological fold, and even many of the phenomena of its life-history, could be imitated by the lateral compression of flexible sheets of material; and how as the pressure increases the original symmetrical undulation be:omes progressively deformed. The fold divides itself ultimately more or less definitely into three parts; the "arch-

No. I 278 , voL. 49 ] 
limb," the "trough-limb," and a central limb of contrary, motion, which is knqwn as the "middle limb" or "septum." In the different regions studied by the geologist, the mode in which this middle limb or septum yields varies greatly according as the material which is being folded behaves as if it were elastic, flexible, or rigid ; the strata in the septum or middle limb being sometimes sheared, sometimes bent, but in the majority of cases becomes twisted and broken, while the parts of the fold move most easily and rapidly in proportion as the septum approaches the perpendicular.

Illustrating the behaviour of this middle limb or septum by the corresponding behaviour of its representative in tidal waves, wind waves, and waves of the sea, \&c., and in flexible and brittle sheets of material, the author pointed out that in all these cases the wave or fold, however much deformed, always consisted in essence of the two reciprocal halves of the arch and the trough; but as it became more compressed, the deformation became more and more concentrated within the middle or septal portion of the fold.

These results not only constitute the key of the geological position, but give us a clue to several of the more remarkable secondary phenomena of the earth's surface, and at the same time afford us a means of grouping together and reducing to fairly natural order many of its supposed anomalies.

From this fresh point of view we now regard the undulations of the earth's surface not only as wave-forms, and consequently each made up of two reciprocal and balanced elements, the one positive and the other negative, but we also look upon them as folds of various degrees of development, all undergoing a progressive deformation.

The recognisable amount of this deformation in any surface fold affords us a rough index of that especial stage in its lifehistory which the foid has attained; and such a fold should present the phenomena characteristic of a typical geological fold at that special stage of its development.

The counterbalance or dissymmetry of the positive and negative parts of the narrower and more continuous earth-folds is well illustrated in the case of the great western marginal ridge of the Americas. The crest of the Rocky Mountain-Andes plateau is the longest, straightest, and most continuous ridge on the face of the globe; and it is bordered throughout, as it should be, by its natural reciprocal - the Eastern Pacific depression or trough, which is correspondingly long, deep, straight, and continuous; and the two together constitute a single crust-fold.

Where, on the other hand, the component crests of the great compound earth-ridges are short, irregular, and confused, the reciprocal compound depressions are correspondingly short and irregular; as, for example, the compound arch of the Alpine ranges, when compared with its compound reciprocal, the Mediterranean troughs.

The same balance of parts of the two component halves of every crust-wave is discernible even in those subordinate examples where, as in the cases of the archipelagoes, the entire wave is almost wholly carried under water in the trough of a larger oceanic wave, for the collective island-arch immediately overlooks its reciprocal-a deep groove in the ocean-floor. Again, where, as in the cases of the Alps and the Himalayas, the sub. ordinate wave is lifted on the back of a grander continental arch completely out of water, its necessary reciprocal or depression, which at first glance appears to be presumably absent, is found by the geologist to be tucked in in the form of a buried valley, for miles below the great mountain ridge, which has been forced forward, beyond, and above it.

The same rule holds good even when the collective dry-land areas are regarded as constituting a single arch. Where the marginal septal zone of this continental arch dies down insensibly towards the North Pole, we have the shallow reciprocal basin of the Arctic Ocean; but upon the opposite edge of the arch, where the septal slope rises up steeply and boldly, as along the outer and higher and shattered rim of the continents facing the Pacific and Indian Oceans, the grandly elevated but broken crest, the continental wave looks out immediately, as theoretically it should do, over its negative reciprocal the most greatly depressed and broken parts of the ocean-floor.

In the case of the geological fold, the study of its life-history shows that the region of yielding and fracture is of necessity the middle region or septum, and that the folding movement takes place most swiftly and easily as this septal portion increases in steepness.

These natural phenomena of the fold we also find paralleled in the case of the earth-waves, whether major or minor. The septal areas and lines dividing the two component halves of the great earth-surface folds mark out distinctly the areas and lines of maximum present volcanicity and earthquake movement on the face of the globe. In proportion as the septal slopes are well marked, long, steep, and continuous, or vice versa, so the intensity of crust-movement and vulcanicity seem to vary from region to region.

The septal area of the seaward edge of the great RockyMountain-Andes fold is not only the septum of the longest and most continuous crust-fold of the present day, but it actually constitutes the longest and most continuous line of present volcanic and earthquake action. The steep outward septal edge of the collective continental mass of the globe, sweeping from Behring Strait to the East Indies, thence to the Cape of Good Hope and Cape Horn back to Behring Strait, shows from end to end its littoral or submarine volcanoes; while the almost in. sensible septal edge of the collective continental arch facing the shallow Arctic depressions, shows not a single volcano along the gentle septal declivity for the whole of its extent. In obedience to the same law, surface land marking the steeper edges of all (or, in other words, their septal slopes) of the great mountain plateaus of the Old World, where they face their reciprocals (the deeper plains in front of them), from the Bay of Bengal through the Himalayas, Hindoo Koosh to the Alps and the Mediterranean shores, constitutes the most active and typical zone of continental earthquakes. This rule of septal yielding and movement not only obtains when the great earth-surface waves are regarded in section, but also when they are figured in plan.

The great compound trough or basin of the Pacific shows all along its septal edge dividing it from its reciprocal or complement, namely, the higher parts of the earth's surface which bound it, an almost complete ring of active volcanoes; and when a projection is made of the entire earth's superficies, having the North Pole as its centre, it is found that this long volcanic band of the Pacific practically divides that surface in two. It is the primary septal band of the earth's superficies, ranging twice from pole to pole.

It was next shown that the minor local surface wrinkles of the earth-crust are not only folds in section, and domes and basins when seen in plan, but that they comport themselves as folds even when regarded laterally or horizontally; the line marking the axes of their crests creeping or flowing horizontally outwards and forwards towards the reciprocal deeps in front. In this way the festoon islands which margin the Pacific, and also the outwardly cnrving shores of the continents, find an additional explanation.

Finally, it was pointed out that if the theory of the fundamental character and domination of the fold or wave in the forms of the earth's surface be well founded, it must necessarily include the most conspicuous features of the earth's surfacerelief regarded as a whole. The existence of this paramount feature was first made known to us by the recent deep-sea researches, which made it evident that the vertical relief of the earth-surface regarded collectively consisted of two membersnamely, the so-called Continental Plateau (of which our present lands are merely the unsubmerged portions), and the so.called Abyssal Region, 12,000 feet and more in depth; these two contrasted elements being united normally by a rapid transitional slope, which lies buried from sight, at a depth of from IO00 to 2000 fathoms below the sea-level.

This remarkable phenomenon the author now interpreted as perfectly natural, and indeed inevitable upon the theory of the crust-fold. The Continental Plateau is merely the collective arch (or dome) of the entire relief of the globe, and the Abyssal Region is the collective trough (or basin), while the intermediate slope is merely the natural septal slope common to the two. But, of course, if this view is correct, it follows of necessity, from the characteristics of a fold (I) that the line marking the position of the axial horizontal plane separating the great earth-arch from the great earth-trough must be about midway down this septal slope; (2) the entire area of the surface of the dome must be equal to that of the basin; (3) the collective volume of the dome must be equal to that of the basin; and (4) that wherever the septal slope is fairly straight it must coincide in direction with the nodal lines of the earth's surface.

It was pointed out by the author that it had already been satisfactorily demonstrated by the results of the calculations and

No. 1278 , voL. 49] 
researches of Mr. Murray, Dr. H. R. Mill, and others, that al these necessary correlations actually existed, although hitherto some of them had been looked upon as mere curious and inexplicable coincidences.

But if the fold or wave rules in the arrangement of the forms of the earth-surface of the present day, it must of necessity rule also in corresponding planetary surfaces, both in space and time; and the author gave it as his opinion that it afforded an equally natural and plausible explanation of cycles, systems, and transgressions of the geological formations, and of the surface (for example) of the planet Mars.

The final conclusion which the author drew from a consideration of the known facts and phenomena was, that the wave or fold appeared to be the natural unit of classification of all the grander forms of the earth-surface. The recognisable surface undulations of the present earth-surface are, broadly speaking, the surfaces of corresponding waves or warpings of the outer parts of the earth-crust, in part obliterated by erosion, \&c., and in part masked by deposition. In the crust-wave, its divisions, modifications, combinations, and intersections, we seem to find the key to the dissymmetries, the harmonies, the contrasts, and even the supposed anomalies of the surface features of the globe. Upon the surface of the earth, the crust-deformation expressible in terms of this unit seems to be the paramount factor. Denudation, deposition, earthquake movement, volcanicity, and even the surface forms and aistributions of the main land and water areas, appear to be all subordinated to this ruling element. As the minor undulations stand related to the major undulations as subordinates, it is probable that not the slightest local change can be brought about without disturbing to that extent the balance of parts, and so leading to a readjustment of the equilibrium of the whole. The fold theory, however, affords us merely a natural and convenient means of classification of surface form, and in the meantime does not concern itself with the mode of origin of these forms. It is a theory, not of causes, but of the most natural grouping of effects.

\section{SCIENTIFIC SERIALS}

American Fournal of Science, April.-Further studies of the drainage features of the Upper Ohio basin, by T. C. Chamberlin and Frank Leverett. The general view adopted is that of Carll, according to whom the present drainage system of the Upper Ohio basin has been formed by the union of several pre-glacial systems that formerly flowed into what is now the Lake Erie basin. These were blocked up by the ice of the earlier glacial period, which invaded their lower courses and forced them to flow over low divides and unite to form a common south-westward flowing system nearly parallel to the border of the ice. The evidence for reversals and displacements of river beds is given in detail, and four hypotheses are presented to account for them. They all greatly emphasise the importance of the first glacial epoch, and indicate that, while the last glacial invasion was very much more pronounced in its apparent effects, it was, after all, much the smaller factor in the glacial period. An apparatus to show, simultaneously to several hearers, the blending of the sensations of interrupted tones, by Alfred $M$. Mayer. A short brass tube is cemented in a hole in the bottom of a glass flask. When the tube is closed the flask resounds powerfully to a tuning-fork of suitable pitch vibrating near its mouth. When the tube is open the resonance is very feeble. The opening and closing is effected by a perforated disc rotating in contact with the brass tube. At a certain velocity the in terrupted sounds blend into the sound of the tuning-fork, the velocity giving an indication of the amount of residual sensation. -The appendages of the pygidium of Triarthrus, by Charles E. Beecher. Further studies of the Yale Museum specimens have enabled the author to make out the main characteristics of the appendages of the caudal shield. At the pygidium, the endopodites preserve the slender, jointed, distal portion found at the thorax, but the proximal part is composed of segments which are considerably expanded transversely, thus making a paddle-like organ, the anterior edge of which is straight, while the posterior one is serrated by the projecting points of the expanded segments. These points bear small bundles of setæ. The specimens from which these details are gathered are very perfectly preserved. The author proposes next to describe the structure of the under side of the head, and then to review the present enlarged knowledge of Triarthrus, with its bearings upon the position and affinities of the Trilobites generally.

Bulletin de l'Académie Royale de Belgique, No. 2. - The sense and the period of the Eulerian movement, by F. Folie. The sense of the Eulerian movement of the pole of inertia round the instantaneous pole is direct; that of the movement of the in stantaneous pole at the surface of the earth is retrograde. The period of the latter is $32 \mathrm{I}$ days; for an integral number of years, a direct and somewhat slower motion may be substituted for this, giving the commonly accepted period of 423 days. But the shorter period is free from the geometrical objections attached to the latter. - The influence of pressure upon specific heat, taken below and above the critical temperature, by P. de Heen. The law governing this influence is analogous to that determining the relation between pressure and compressibility. Little variable at first, the specific heat rises with increasing pressure up to a certain limit, and then diminishes. - On the phenomenon of beats in luminous vibrations, by Dr. J Verschaffelt. Prof. Righi showed in 1878 that if two rays are brought to interference whose periods are only slightly different, fringes are obtained which move with such velocity that a number equal to the difference of frequency passes each point of the screen in one second. Righi realised this practically by means of a rotating Nicoll prism and Fresnel's mirror. The principle applied by Dr. Verschaffelt is that of Doppler, according to which a motion of the source whth respect to the ether changes the wave-length of the light emitted. The retardation was produced by a moving wedge of quartz cut parallel to its axis, and placed at $45^{\circ}$ between the crossed Nicolls of a polarising microscope.-On absorption by the bile ducts, by Célestin Tobias. Ligature of the thoracic canal suppresses absorption of acids and biliary pigments, as pointed out by Harley. But it does not affect that of sodium ferrocyanide, of strychnine, or of atropine at the surface of the bile ducts. Sodium iodide is not absorbed at all. Whether the absorption is lymphatic or sanguine depends upon the nature of the substance.

\section{SOCIETIES AND ACADEMIES.} LONDON.

Physical Society, April 13.-Prof. A. W. Riicker, F.R.S., President, in the chair. - The President invited discussion on Prof. Henrici's paper on calculating machines, and said a description of Mr. Sharp's harmonic analyser, giving direct readings of the amplitude and epoch of the various constituent simple harmonic terms, had been sent in. This machine requires no adjustments to be made before using. The amplitude is given by the length of a line joining the initial and final positions of the point of contact of a roller with a rotating disc, whilst the epoch is determined by the angle which this line makes with the plane of the roller in its initial position. - Prof. Perry congratulated Prof. Henrici on the success attained with his analysers. Referring to planimeters, he said the average error made in working out indicator diagrams with Hine and Robertson's instrument was only about one-third that made with Amsler's. After pointing out the great importance of Fourier's series to practical men, and especially to electrical engineers, he said that in studying reciprocating motions, such as those of pistons, valve gears, \&c. it was most useful to resolve the motion into its fundamental harmonic motions and its overtones. In this way remarkable differences could be seen between various motions which have the same fundamental, and which are usually considered equivalent. In the Electrician of February 5, 1892, he had published the numerical work for a given periodic curve developed in Fourier's series, and he now exhibited a graphical solution done by one of his students, who was probably the first to carry out the late Prof. Clifford's idea of wrapping the curve round a cylinder and projecting it on different planes. Prof. Henrici had, he said, based the construction of his first analyser on Clifford's method, but used the Henrici principle (viz. $\int y \sin \theta d \theta=\int \cos \theta d y$, when integrated over a complete period) to explain the later machines. As a matter of fact the first machine in which the coefficients were determined by an Amsler planimeter carried by a reciprocating tangent plane, was a beautiful example of the Henrici principle, and he, Prof. Perry, saw far greater possibilities before it. The defects in the first instrument were mechanical ones, and could be got over by in-

NO. I 278 , VOL, 49 | 